\title{
DEL ARCO AL EJE MEDITERRÁNEO EN ESPAÑA HACIA LA CONSOLIDACIÓN DE UN CORREDOR DE TRANSPORTES
}

JOSÉ Ma . SERRANO MARTÍNEZ ${ }^{1}$

\begin{abstract}
Resumen - Durante las últimas décadas las áreas próximas al Arco Mediterráneo español han tenido un rápido e intenso incremento demográfico, aumento de los espacios urbanizados y crecimiento de su actividad productiva diversificada. Lo cual ha contribuido a generar importantes movimientos de tráfico. La carretera es el principal modo de transporte que encauza los mayores flujos. Ahora, se pretende completar las infraestructuras de transportes a través de ambiciosos proyectos de inversión en ferrocarriles. Eso consolidaría su papel de Corredor, sentándose las bases para la futura organización de un Eje de desarrollo. Se aprovecha su buena disposición geoestratégica dentro del marco europeo Mediterráneo para alcanzar un Eje de desarrollo. Se buscan sus ventajas de localización en referencia a los pujantes espacios del mundo. También se reflexiona acerca de lo que ello puede representar dentro de una organización equilibrada territorial del conjunto español, ibérico y su vertebración espacial.
\end{abstract}

Palabras clave: Transportes en el área mediterránea, infraestructuras y desarrollo, organización del territorio.

Resumo - DO ARCO AO EIXO MEDITERRÂNEO (ESPANHA), ATÉ À CONSOLIDAÇÃO DE UM CORREDOR DE TRANSPORTES. Nas últimas décadas assistiu-se a uma evolução positiva nas regiões próximas ao Arco Mediterrâneo. Esse facto traduziu-se no aumento dos valores demográficos, no incremento da urbanização e no crescimento e diversificação da respectiva actividade produtiva, factos que têm contribuído para o incremento do tráfico. Destes movimentos, a rodovia é a infra-estrutura que acolhe fluxos mais intensos. Contudo, na actualidade pretendem-se diversificar estas redes através de projectos ambiciosos no domínio das ferrovias. Esta aposta reforçaria o papel de Corredor desempenhado por esta região, facto importante para consolidar as bases da futura organização de um eixo de desenvolvimento. Este tiraria partido da boa localização geoestratégica deste território no contexto da Europa do Mediterrâneo, vantagem locativa que terá também como alvo outros espaços dinâmicos.

Palavras-chave: Transportes na região mediterrânea, infra-estruturas e desenvolvimento, organização territorial.

Recebido: Novembro 2011. Aceite: Maio 2012.

1 Departamento de Geografía. Campus La Merced, Universidad de Murcia, España.

E-mail: jmserran@um.es 


\begin{abstract}
From the arC to the MediterRanean aXis In SPAin, towards the CONSOLIDATION OF A TRANSPORTATION CORRIDOR. There has been a significant development in the regions of the Mediterranean Arc in recent decades. This fact has led to raised demographic values, a boost in urbanization and to the growth and diversification of its productive activity, which in turn have contributed to an increase in traffic. The highway is the main transportation way and it channels the heaviest traffic flows. However, there are plans in place to diversify these transportation networks through ambitious investment projects in railways, which would consolidate the role of Corridor played by this region in laying down the foundations for the future organization of an Axis of development. This would take advantage of the geoestrategic location of this territory, in the context of Mediterranean Europe, to target other dynamic spaces.
\end{abstract}

Keywords: Transport, Mediterranean area, infrastructures, development, organization of the territory.

Résumé - DE L'ARC À L'AXE MÉDITERRANÉEN EN ESPAGNE, JUSQU'À LA CONSOLIDATION D'UN CORRIDOR DE TRANSPORT. Durant les dernières décennies, les zones proches de l'Arc méditerranéen espagnol ont connu une croissance démographique prompte et intense, une augmentation sensible des zones urbaines, ainsi qu'un accroissement et une diversification de leurs activités productives, ce qui a contribué à une densification du trafic. La route est l'infrastructure qui accueille les flux les plus importants. Cependant, on essaie aujourd'hui de diversifier les infrastructures de transport à travers d'ambitieux projets d'investissements ferroviaires. Cela renforcerait le rôle de Corridor principal joué par cette région, consolidant ainsi les bases de l'organisation future d'un axe de développement, qui bénéficierait de son avantageuse localisation géostratégique dans l'Europe méditerranéenne et qui constituerait un élément fort de connexion entre l'Europe et l'Asie. Dans cette perspective, on réfléchit également à l'influence que peut avoir cette politique sur les équilibres de la structuration spatiale de l'Espagne et de la péninsule Ibérique.

Mots-clés: Transports dans la zone méditerranéenne, infrastructures et développement, organisation du territoire.

\title{
I. PRESENTACIÓN Y CONSIDERACIONES BÁSICAS
}

En esencia, los transportes constituyen un sector intermedio en la actividad económica, son básicos en el ciclo productivo. De ahí su particular significación. Debe considerarse, en primer termino, su profunda interrelación con el modelo de desarrollo territorial y las formaciones sociales que caracterizan toda unidad espacial. Ahora bien, al mismo tiempo el sistema de transportes define un conjunto de potenciales del territorio. Se vinculan a la dispar evolución de sus actividades. De manera básica desempeñan un triple papel:

- Dotan de accesibilidad al territorio; es decir, permiten que a cada punto del mismo alcancen los distintos agentes sociales que interactúan en él, definiendo potenciales de acceso, directamente ligados al concepto de competitividad territorial.

- Facilitan la conexión económica, tanto horizontal como vertical.

- Ofrecen y posibilitan la realización del transporte como actividad productiva. 
Además, el desarrollo del transporte encierra una doble tarea complementaria (Chesnais, 1997). De un lado, en su fase inicial se precisa la construcción y puesta en servicio de las infraestructuras pertinentes; merced a inversiones, a menudo, cuantiosas. De otro, su funcionamiento cotidiano, necesita alcanzar la operatividad suficiente para que, costes y beneficios, no caminen de forma divergente. Por ello, la complejidad de facetas inherentes y derivadas de la puesta en servicio y funcionamiento de los transportes es muy grande (Merlin, 1991). Su dimensión es plural, con comportamientos derivados en cascada. Así, una parte, a menudo sustancial, de las consecuencias derivadas de la construcción de infraestructuras, resulta difícil de valorar (Cuadrado, 1992: 63). Pero, lo cierto es que tales efectos existen y pueden llegar a ser decisivos para un área o territorio concreto; tanto por su dimensión, como por su oportunidad.

En esta investigación el problema básico abordado es: en el presente (2012) no existe continuidad adecuada en los trazados de infraestructuras terrestres (sobre todo, en ferrocarril), que permitan la circulación de personas y mercancías a lo largo del Arco Mediterráneo español. Las instalaciones portuarias y logísticas existentes padecen la dificultad de sus conexiones. La accesibilidad entre las diferentes y numerosas aglomeraciones urbanas y áreas metropolitanas, ubicadas en él, se diluye en cuanto se traspasan sus inmediatas áreas funcionales (Fernández et al., 1992). Su comportamiento se asemeja a un archipiélago fragmentado, a veces denso, más que a un corredor; dista mucho de ser un Eje. Tal realidad interna también añade serias dificultades exteriores. Son manifiestos los inconvenientes en su conexión exterior, tanto en su prolongación con el Arco Latino (hacia Francia e Italia), como en sus engarces con la dorsal europea, a través del valle del Ródano.

Talvez se han desaprovechado en España los años cuando se han realizado copiosas inversiones en infraestructuras de transportes (con fuertes ayudas Comunitarias), no siempre tan prioritarias, como pueden ser éstas, para mejorarlo. Ahora el panorama es más complejo. Recientemente, se ha seleccionado, el citado Arco Mediterráneo, (X-2011 y III-2012) como eje vertebrador de ámbito europeo; si bien de manera más completa sólo en su parte Norte. Por ello, parece oportuno analizar los numerosos aspectos relacionados con ello. También, su papel dentro del modelo territorial español global, con referencia a un marco Ibérico más extenso (Portugal). Se pretende, pues, calibrar su función en el deseable equilibrio espacial de España, dentro del horizonte de una mayor integración peninsular. Todos esos proyectos no se deben enfocar como un fin en si mismos, sino como un medio. De lo contrario, estaríamos repitiendo el pasado modelo económico, donde la inversión en "obra pública" se convirtió, a menudo, en elemento esencial y motor básico del sistema productivo nacional (Fernández, 2006). Ahora, (desde 2007) se sufren las consecuencias de los excesos anteriores.

Abordar un tema tan vasto e interesante precisa hacerlo con metodología adecuada. Se parte de los procedimientos habituales de investigación histórica, teniendo en cuenta lo que otros estudiosos han investigado sobre el asunto. Esto resulta com- 
plejo por ser un tema pluridisciplinar, objeto de atención desde varios campos científicos: donde concurren economistas, geógrafos y otros profesionales preocupados por la ordenación del territorio. Eso añade dificultades adicionales, dada la pluralidad de puntos de vista utilizados. También, es necesario combinar el análisis y la síntesis geográfica, para, de esa manera, acoplar las aplicaciones de escala. En algunos apartados es necesario contemplar el conjunto territorial español, e incluso con una visión más amplia, toda la Unión Europea. En otros, interesa focalizar el detalle en áreas específicas del marco Mediterráneo, para desentrañar aspectos singulares y trascendentes. El uso de abundante información estadística se subsana con la confección de cuadros, resumiendo los valores más destacados y significativos. La aportación de tres figuras cartográficas ayuda a su exposición sintética y clara; así se evita un análisis escrito más detallado.

El desarrollo de la investigación se realiza a través de tres apartados básicos: En el primero se aborda la interpretación esencial del Arco Mediterráneo español. En el siguiente se replantea una discusión sobre el tema central; y en el último se analiza la vertebración nacional y el papel que dentro de ella puede desempeñar ese futuro, y probable, Eje Mediterráneo.

Interesa añadir unas precisiones terminológicas. A menudo se utilizan varios vocablos de manera indistinta, al referirse al ámbito objeto de este estudio. Aquí se propone hacerlo con la siguiente matización: Arco Mediterráneo es la franja litoral mediterránea, en referencia concreta a su disposición general y a su continuidad espacial. Corredor Mediterráneo, implica la existencia (en ese ámbito) de abundantes flujos de transporte y relación, combinados, entre todo el territorio. Eje Mediterráneo, sería el espacio integrado y vertebrado por la combinación de plurales relaciones económicas y productivas, que conllevan un funcionamiento conjunto e interdependiente intenso. Con esa propuesta sobre términos, estaríamos ante fases y situaciones sucesivas (Seguí \& Martínez, 2004). Se parte de una inicial realidad morfológica, física, a otra de clara intervención antrópica y de ordenación del territorio.

\section{RASGOS COMPLEJOS Y HETEROGÉNEOS DEL ARCO MEDITERRÁNEO ESPAÑOL}

El litoral mediterráneo español peninsular se extiende por casi dos mil Km. (1861 Km.) de costa. Corresponde a 11 provincias que, a su vez, se engloban en cuatro Comunidades autónomas (Andalucía, Murcia, Comunidad de Valencia y Cataluña). La superficie de esas provincias suma $90.490 \mathrm{~km} 2$ (el 17,2 \% del conjunto español). Su disposición muestra dos grandes arcos; el primero, desde Cádiz a Almería; el segundo se organiza, a su vez, en tres porciones menores: entre Cabo de Gata y Palos; de éste a Cabo de la Nao (Alicante); y, a continuación, con costas más rectilíneas, se alcanza el Cabo de Creus, próximo a la frontera francesa. La topografía del territorio es variada, con fuertes contrastes. Entre Algeciras (Cádiz) y Murcia- 
-Alicante, las alineaciones montañosas se aproximan a la costa, dejando estrechas y separadas llanuras litorales. En el resto, hacia el Norte, la anchura de las planicies litorales es mayor, si bien interrumpidas por ciertos plegamientos que caen directamente sobre la costa. Así las áreas llanas y de escasa elevación (inferior a los 200 metros de altura) representan proporciones contrastadas. Algunos datos escuetos y complementarios, se detallan en el cuadro 1.

Cuadro I - Rasgos básicos del territorio.

Table I-Basic features of the territory.

\begin{tabular}{lcccc}
\hline & $\mathrm{h} / \mathrm{km}^{2}$ & Extensión, $\mathrm{km}^{2}$ & $\%<200 \mathrm{~m}$ altitud & $\begin{array}{c}\mathrm{km} \\
\text { litoral }\end{array}$ \\
\hline Cádiz & 167,4 & 7385 & 69,66 & 252 \\
Málaga & 221,2 & 7276 & 17,64 & 161 \\
Granada & 73,2 & 12531 & 1,61 & 71 \\
Almería & 79,2 & 8774 & 8,93 & 217 \\
Murcia & 129,1 & 11317 & 14,95 & 208 \\
Alicante & 328,5 & 5863 & 36,90 & 212 \\
Valencia & 239,8 & 10763 & 25,40 & 109 \\
Castellón & 90,4 & 6679 & 18,02 & 116 \\
Tarragona & 128,6 & 6283 & 36,60 & 216 \\
Barcelona & 712,6 & 7733 & 17,65 & 101 \\
Gerona & 127,9 & 5886 & 41,16 & 198 \\
\hline Media, área & 200,0 & 90490 & 21,16 & 1861 \\
\hline Total España & 93,1 & 504750 & $* 11,37$ & $* * * 3904$ \\
\hline \% área/España & \multicolumn{5}{c}{$* 3,53,53$} & 47,66 \\
\hline
\end{tabular}

Fuente: Elaboración propia sobre datos del I. N. E.

*promedio nacional (superficie bajo $200 \mathrm{~m}$.), **proporción de territorio del área bajo 200 metros, sobre el total,

$* * *$ costa peninsular (asciende a $2.217 \mathrm{~km}$ ).

Tras esa breve presentación interesa destacar ciertos rasgos básicos para interpretar su potencial territorial y su accesibilidad: Sus condicionantes naturales, topográficos y climáticos, han favorecido una agricultura especializada e intensiva, desde tiempo, renovada continuamente. Las actividades industriales se distribuyen de manera muy contrastada en él. Más de medio siglo de desarrollo turístico constituye uno de sus activos económicos básicos en el presente. Más adelante, se volverá sobre todo esto.

Para interpretar el asunto central de esta investigación (el camino de consolidación, hacia el referido Eje Mediterráneo), interesa considerar ciertos aspectos demográficos; centrados en los años más recientes. En 2001 (censo), su población ascendía a 15,1 millones de personas (36,9 \% del conjunto español). En 2011 (padrón continuo) registraba 18,1 millones $(38,4 \%$, respectivamente). Eso confirma un apreciable dinamismo poblacional: aumento neto de 3 millones de residentes y ascenso de su significación porcentual de casi dos puntos. Igualmente. Su densidad media de 
población es superior a la española: $200 \mathrm{~h} / \mathrm{m} 2$ frente a 93,1. La inmigración extranjera ha sido causa básica de ese ascenso poblacional reciente.

A su vez, las disparidades demográficas internas son acusadas, en datos absolutos y relativos. La debilidad demográfica de Granada y Almería se contrapone la de Barcelona, Valencia, Alicante o Málaga. Tales contrastes adquieren mayor intensidad al descender en análisis de escalas más detalladas. Así, junto a áreas de fuerte concentración, existen vacíos demográficos. La orografía constituye un punto de partida para interpretar la ocupación humana. Sin embargo, sus efectos han variado al paso del tiempo: a la mayor densidad demográfica tradicional de la montaña, sucede el progresivo protagonismo del litoral. Nuevas actividades y nuevas formas de vida, conllevan modelos e intensidades de ocupación diferenciadas.

Las significativas disparidades demográficas actuales internas, obedecen a pluralidad de causas. Unas inmediatas, precipitadas hace años por los complejos flujos migratorios interiores (en sus diferentes escalas locales, comarcales, provinciales, intrarregionales e interregionales) y exteriores. Otras mediatas, son consecuencia de la evolución de procesos socioeconómicos diferentes a través de los cuales, las provincias costeras, y sobre todo, las comarcas y áreas más vecinas al litoral, registraron balances productivos más favorables, atrayendo a la población. Las actividades ligadas al turismo, tiempo libre, ocio y recreación merecen atención destacada, por sus efectos derivados, desde mediados del siglo pasado (Alcaide, 1988, Pedreño, 1988). Ciertas precisiones al respecto permiten entender su estructura productiva y significación dentro del conjunto español.

Los datos totales del PIB confirman tal afirmación (su valor absoluto se cifra en 386 mil millones de $€$ en 2009). Sin embargo, su proporción sobre el total nacional es inferior a la alcanzada por sus valores demográficos (-1,89 puntos). L propia heterogeneidad territorial arrastra a la baja los valores medios. Junto a un PIB per cápita más elevado en algunas provincias: Gerona, Castellón, Barcelona, otras registran cifras reducidas: Granada, Cádiz, etc. Así, las diferencias internas absolutas son acusadas: ascienden a 11,3 veces (Almería/Barcelona). Mayores que las derivadas de su peso demográfico $(8,9)$. Dadas las limitaciones de espacio y al objetivo buscado aquí (interpretación de la realidad territorial, en el campo de los transportes), se precisan sólo unas breves consideraciones relativas a los tres sectores económicos más representativos. Su suma global, significa el 73,83\% del conjunto del PIB de ese ámbito territorial. Interesa fijarse, entre otros, en los siguientes aspectos:

i) El apartado genérico englobado dentro de la denominada "industria transformadora" alcanza mayor proporción que la de su peso poblacional $(4,18$ puntos). Constituye un sesgo sectorial favorable, el mayor de todos. Si bien las diferencias interiores de las provincias incluidas son abultados. Basta comparar las cifras extremas que aportan las provincias de: Barcelona y Almería. Ambas muestran una disparidad de 1/43,9 veces. Ambas registran los mayores contrastes. Son fruto de una situación gestada hace tiempo.

ii) En el sector primario, a pesar de las elevadas cifras aportadas por la citada especialización de las agriculturas tempranas (cuyos casos más representa- 
tivos corresponden a las provincias de Valencia, Murcia, Almería y Valencia), su significación porcentual (sobre el conjunto nacional), es inferior a su aportación demográfica (-2,39 puntos). Constituye, en ese sentido, una falacia simplista, asociar el área mediterránea, en esencia, con la especialización agraria.

iii) Los servicios también contabilizan valores de promedio $1,7 \%$ inferior a su significación poblacional. Aunque las cifras acumuladas son elevadas, no son suficientes para sobrepasar el promedio español. Como epítome de este amplio apartado, constituyen un caso paradigmático las actividades turísticas. De éstas, uno de los indicadores a cotejar, son las plazas hoteleras. Se contabilizan en total 632.606. Valor absoluto elevado, sin duda. Sin embargo, en proporción, representa 2,01 puntos menos de su significación demográfica. Al quedar fuera los dos archipiélagos (más cercano, en este caso, Baleares) donde se concentran contingentes elevados de esos servicios, se aminora su significación global.

En resumen, pues, la significación de estas provincias, a pesar de su reducida extensión, concentra valores elevados en sus efectivos humanos y significativos en otros apartados productivos y económicos. Todo ello conlleva importantes cantidades de producción, con sus correspondientes entradas y salidas de mercancías; lo cual precisa una red densa de infraestructuras de transporte, para permitir la elevada movilidad y desplazamientos. La disposición longitudinal del corredor concentra los flujos de tráfico, tanto interiores como de tránsito, tanto españoles como de sus áreas vecinas (desde Marruecos a Francia), a través de sus viales básicos terrestres, sean de carretera o de ferrocarril.

Otro aspecto importante de interpretación tiene que ver con los rasgos singulares de su disposición poblacional y urbana. Interesa fijarse en especial sobre esta última. Debe hacerse hincapié en el grado de urbanización existente y en la singularidad de organización de la malla urbana. Las ciudades siempre constituyen centros básicos de actividad productiva. Son los nodos claves para vertebrar el territorio (Serrano, 2008). Cualquier análisis y toda proyección de futuro de los sistemas de transporte, sólo pueden enfocarse desde la óptica que ofrece su realidad urbana. La existencia de nodos, la disposición de redes urbanas y la organización de subsistemas de ciudades, conforman un apartado esencial, en rápido cambio, desde hace años (Vegara, 1992). Eso influye en la propia organización del Arco, en la posterior estructuración del Corredor y, en su caso, en la futura consolidación del Eje Mediterráneo. Aquí, el promedio de urbanización es superior al español (se alcanza el $83 \%$ ). La red de ciudades es densa, configura una trama completa. Se organiza, en esencia, desde las ciudades capitales de provincia. Pero, más bien, hay que hablar de una serie de aglomeraciones urbanas (en adelante AU) como principales centros de concentración y polarización territorial. Para resumir en extremo el asunto, la figura 1 permite contemplar su dimensión y esquema territorial. $\mathrm{Su}$ disposición y jerarquía ayudan a comprender el funcionamiento de este ámbito mediterráneo. 


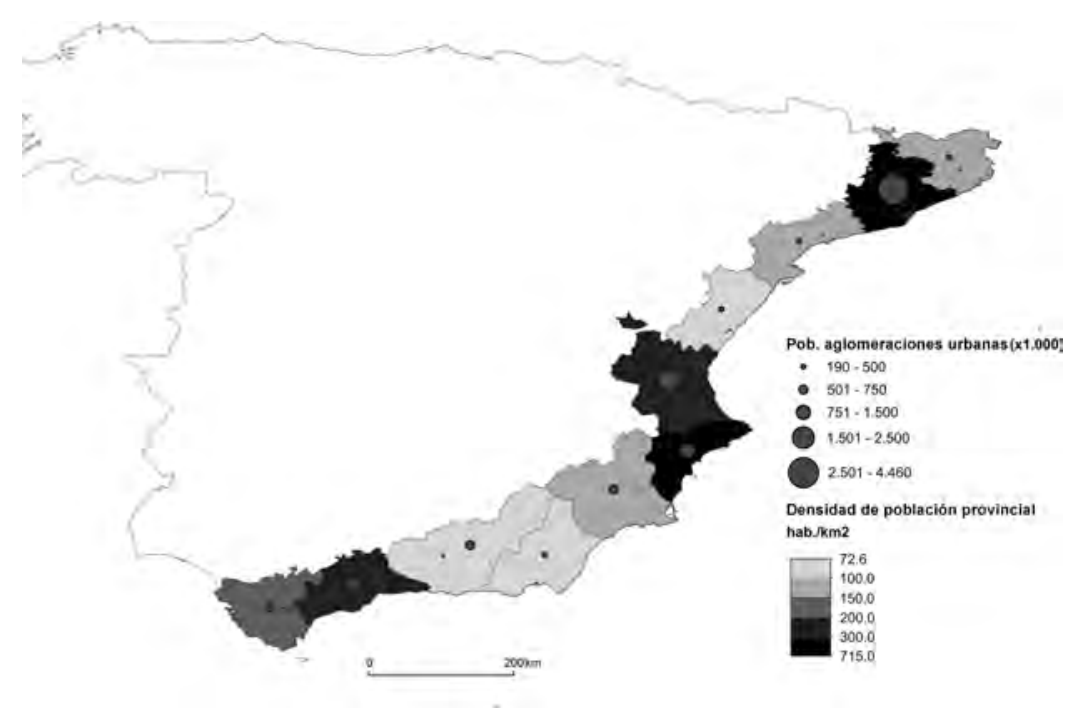

Fig. 1 - Aglomeraciones urbanas y capitales de provincia.

Fig. 1 - Urban agglomerations and provincial capitals.

\section{ACCESIBILIDAD Y COMUNICACIÓN A TRAVÉS DEL CORREDOR MEDITERRÁNEO}

Se añaden unas breves consideraciones acerca de los flujos de tráfico a través de los diferentes modos de transporte; la figura 2 ayuda ello. Se busca, a través de la disposición de los trazados de infraestructuras, calibrar los mayores acomodos y desajustes existentes.

Dentro del modo terrestre, las infraestructuras de carreteras son las más densas y complejas. La mayor capilaridad en su trazado reduce la ruptura de carga, accediendo a sitios recónditos y apartados (Bellet et al., 2010). Aquí sólo es posible hacer referencia a las de mayor relevancia y continuidad de recorrido. Es decir a las autopistas y autovías (en adelante y para abreviar, todas se denominan vías rápidas de gran capacidad, VRGC); se añaden aquellas otras vías principales (en sus tramos básicos), por lo común, vías nacionales, con similares cometidos.

En la mayor parte del Arco Mediterráneo, la AP7 (casi mitad autopista de peaje, el resto autovía de uso libre) discurre junto a la línea de costa. Es el eje viario esencial. En ciertos trechos, existe otra autovía complementaria, más al interior, aprovechando los surcos intrabéticos, que facilitan su trazado, conectando las principales áreas urbanas que conforman el armazón básico de ciudades. La orografía y la morfología de la trama urbana encajan con los trazados viarios básicos. Una realidad complementada al paso del tiempo. Recientes construcciones de VRGC, de ciertos trechos complementarios, para impulsar las áreas costeras más vírgenes, han dado resultados poco rentables. Ello confirma los riesgos de nuevas actuaciones. 


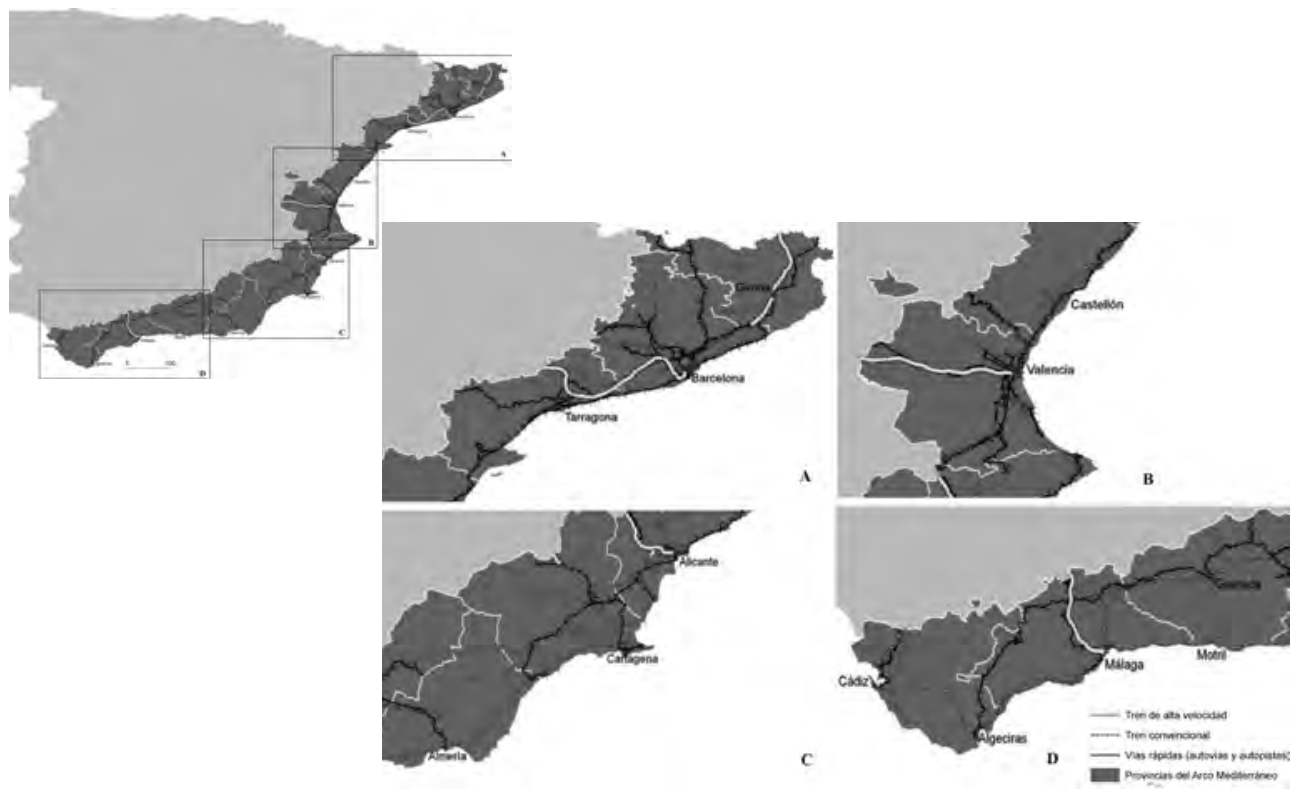

Fig. 2 - Red de infraestructuras terrestres de transporte.

Fig. 2 - Land transport infrastructures network.

De manera global, la carretera acoge, en España, proporciones elevadas de tráfico, de pasajeros y mercancías, en su ámbito interno. Su protagonismo es superior al promedio europeo (Merenne, 1995). En su movimiento interior de viajeros, en 2010 asciende a 90,72 \%; un valor en alza, dado que en 1985 era del 86,90\%. En los desplazamientos internacionales esa cifra es más reducida, en torno al $60 \%$ y con tendencia a encogerse. Por su parte, en lo concerniente a las mercancías en su marco interior, la carretera representa el modo más utilizado; con progresión ascendente. En 1980 era del 68,49\% y en 2010 el 79,64 \%. Sólo en el tráfico internacional de mercancías, la carretera contabiliza proporciones modestas, alrededor de la cuarta parte del total (pero, con horizonte de incremento, $24,92 \%$ en 2010, frente a $8,25 \%$ en 1980).

Dentro de ese marco global, interesa fijarse en el tráfico que discurre por el Arco Mediterráneo. Para facilitar esa tarea el cuadro II resume los principales valores.

Los datos analizados corresponden sólo a las dos vías referidas, de mayor continuidad y significación, a lo largo de todo el Arco, la autopista de peaje AP7, la N340 y otros tramos de VRGC que la complementan y extienden sus conexiones en recorridos vecinos. En un estudio con semejante escala espacial no es posible descender en análisis muy detallados. Se incluyen tres registros temporales para comprobar su evolución. Entre 1980 y 2005, se aprecian intensos incrementos en los aforos de tráfico. A menudo las bases iniciales se multiplican entre 4 y 5 . El propio aumento del parque de vehículos fue muy elevado durante esos años. Las nuevas infraestructuras viarias también impulsan el incremento de la circulación. El ascenso 
de la actividad económica y del nivel de vida fue determinante en ello. Los aforos, sin embargo, no mantienen similares proporciones de aumento en todos los tramos; hay balances dispares. Los datos más recientes, 2010, tienen por objetivo comprobar cómo ha incidido en este apartado la situación de fuerte crisis económica que padece la economía española. En general, se advierte un claro estancamiento, en relación a 2005 , frente a los incrementos anteriores.

Cuadro II - Tráfico por carretera, intensidades medias diarias; total vehículos y vehículos pesados. Table II - Road traffic, daily average intensity; total number of vehicles and heavy vehicles.

\begin{tabular}{|c|c|c|c|c|c|c|c|c|c|c|}
\hline & \multirow{2}{*}{\multicolumn{2}{|c|}{$\begin{array}{c}1980 \\
\text { Vía costera }\end{array}$}} & \multicolumn{4}{|c|}{2005} & \multicolumn{4}{|c|}{2010} \\
\hline & & & \multicolumn{2}{|c|}{$\begin{array}{l}\text { Autopista/ } \\
\text { Autovía }\end{array}$} & \multicolumn{2}{|c|}{ C-340 } & \multicolumn{2}{|c|}{$\begin{array}{l}\text { Autopista/ } \\
\text { Auto-vía }\end{array}$} & \multicolumn{2}{|c|}{ C-340 } \\
\hline & Total & $\% *$ & Total & $\%$ & Total & $\%$ & Total & $\%$ & Total & $\%$ \\
\hline Frontera francesa & 5344 & 19 & 30301 & 28 & 10210 & 2 & 28917 & 28 & 10371 & 3 \\
\hline $\begin{array}{l}\text { Límite, entre. Gerona/ } \\
\text { Barcelona }\end{array}$ & 11470 & 15 & 54202 & 22 & 19672 & 20 & 53343 & 19 & 21822 & 16 \\
\hline "Barcelona/Tarragona & 10997 & 21 & 53226 & 18 & 52846 & 19 & 50450 & 19 & 49774 & 17 \\
\hline “Tarragona/Castellón & 10763 & 27 & 19166 & 22 & 14809 & 29 & 17773 & 21 & 14172 & 29 \\
\hline "Castellón/ Valencia & 32034 & 22 & 27402 & 18 & 21256 & 9 & 19632 & 26 & 39947 & 24 \\
\hline "Valencia/Alicante & 17057 & 13 & 24662 & 14 & 15795 & 12 & 20513 & 10 & 21220 & 11 \\
\hline “Alicante/Murcia & 12324 & 18 & 36232 & 23 & 15093 & 15 & 42735 & 19 & 14662 & 13 \\
\hline “Murcia/Granada & 2327 & 24 & 10973 & 25 & -- & -- & 12652 & 18 & -- & -- \\
\hline “Murcia/Almería & 3644 & 23 & 14144 & 31 & -- & -- & 13356 & 22 & -- & -- \\
\hline “Almería/Granada & 3076 & 22 & -- & -- & 13652 & 14 & -- & -- & 14052 & 10 \\
\hline “Granada/Málaga & 3647 & 15 & 18590 & 8 & -- & -- & 17284 & 9 & -- & -- \\
\hline “Málaga/Cádiz & 5199 & 9 & 16550 & 13 & 16609 & 8 & 22221 & 9 & 18068 & 11 \\
\hline "Cádiz (Vejer de la F.) & 6022 & 15 & -- & -- & 13402 & 7 & -- & -- & 14535 & 6 \\
\hline
\end{tabular}

Fuente. Elaboración propia sobre datos del Ministerio de Fomento, Secretaría General de Infraestructuras, Dirección General de Carreteras, Mapas de aforos de tráfico.

Para evitar distorsiones en el análisis de los datos, se han tomado como referencia aforos de tráfico distantes de las mayores aglomeraciones urbanas. De esa manera, se soslaya la acumulación inducida en sus periferias urbanas. Así puede calibrarse de manera más cabal las diferencias de tráfico en sus sucesivos trechos.

Hay contrastes netos en el discurrir de los flujos de tráfico. Los aforos más copiosos corresponden al tramo que conecta Gerona y Tarragona. Aquí la suma de las dos vías eleva las cantidades en torno a los cien mil vehículos diarios. Esto confirma el papel nodal de la AU de Barcelona en ese ámbito. Sin embargo, hacia el Norte, en las proximidades de la frontera francesa, las cifras se reducen a más de la mitad; alrededor de cuarenta mil vehículos.

Hacia el Sur, la intensidad de circulación desciende. Entre Murcia y Castellón, los flujos, sin ser siempre homogéneos, contabilizan cifras que van de los treinta y cinco mil a los cincuenta mil vehículos. Son cantidades inferiores a las del tramo precedente, si bien más elevadas que las del resto de las vías que recorren este Arco. 
Desde Murcia a Algeciras los flujos de tráfico reducen su intensidad de forma apreciable. La bifurcación de las dos autovías, a partir de Puerto Lumbreras, apenas suma la mitad de los flujos registrados al sur del tramo anterior. Sólo, en su ámbito final, entre Cádiz y Málaga, se contabilizan valores algo mayores, en torno a los cuarenta mil vehículos en 2010 .

Por su parte, los registros correspondientes a los vehículos pesados contienen información de gran interés. De un lado, se comprueba que las proporciones correspondientes al primer año de referencia, 1980, ya contabilizaba cifras en torno al 20 $\%$ en la mayor parte de los tramos. Lo cual corrobora la importancia del tráfico de mercancías, a los cuales se pueden asociar, en gran medida, los denominados vehículos pesados. En años recientes, 2005 y 2010, aún habiéndose multiplicado las intensidades, la proporción de los vehículos pesados mantiene proporciones semejantes. Esos datos confirman que por el conjunto del Arco Mediterráneo, las principales VRGC encauzan una parte sustancial del tráfico pesado, compuesto por mercancías; dentro de un modelo de transporte donde prima la carretera. Numerosas externalidades negativas se desprenden de ello (caso de la contaminación ambiental, acústica, etc.). También, sus limitaciones de futuro son manifiestas en semejante predominio modal (Ortúzar y Willunsen, 2008).

Los transportes terrestres por ferrocarril registran un balance muy distinto a la carretera. Una espiral negativa se abrió hace tiempo. La debilidad y las rupturas de la trama ferroviaria explican su escasa circulación. Los promedios de uso españoles son inferiores a los europeos (Auphan, 1997, C.C.E., 2003, Rodríguez et al., 2006). Unas apretadas referencias a ello se añaden a continuación.

Sólo en el tramo más septentrional, entre la frontera francesa y Valencia, el discurrir del trazado ferroviario circula junto a la costa, con doble vía electrificada, de ancho ibérico (además de la reciente adaptación del último trecho, Barcelonafrontera francesa a la vía europea, terminada en abril de 2011). Hacía el Sur, la conexión con Alicante cuenta con características técnicas parecidas a las antes indicadas, si bien con algún rodeo hacia el interior, por La Encina. Entre Alicante y Murcia sólo hay vía única, sin electrificar, situación que se prolonga hasta Lorca, con dos trechos de enlaces costeros. La ruptura es total hacia el Sur. Dejó de funcionar (enero de 1986) el enlace entre Murcia y Granada por el Valle del Almanzora, dentro de la dinámica de declive sufrida por el ferrocarril en España en el transcurso del último medio siglo. De nuevo, el engarce entre Almería y Málaga, se hace por el trazado tradicional interior (a través de Moreda, Granada y Bobadilla). El trecho de la Costa del Sol carece de trazado ferroviario, salvo un pequeño tramo con tráfico de pasajeros, de naturaleza casi urbana.

Con esa disposición y características no puede hablarse de Corredor Mediterráneo de circulación ferroviaria. Sólo funciona, parcialmente, en su primera parte. Desde esa latitud, hacia el Sur, los escasos trazados ferroviarios en uso se asemejan a apéndices que conectan puntos concretos del litoral (de sus ciudades más significativas), con el centro del sistema ferroviario nacional, Madrid; pero, sin vinculación interna entre ellos. Por eso, no es adecuado calificar la trama viaria existente como red 
ferroviaria, en su parte meridional litoral. En gran medida, la disposición de las infraestructuras casi mantiene los trazados realizados en el siglo XIX (Wais, 1974). Las principales ciudades, áreas urbanas y aglomeraciones metropolitanas desarrolladas junto a la costa, o en espacios cercanos al litoral, carecen de alternativa viable, en sus conexiones internas, a través del ferrocarril. Así, todo enlace entre Málaga-Costa del Sol, AU de Murcia, AU de Alicante y AU de Valencia, o bien se lleva a cabo mediante otros modos de transporte, o se efectúa, realizando un gran rodeo, a través de Madrid. Eso supone mayor recorrido, gasto económico y aumento del tiempo de viaje. Se desaprovecha el papel que podría desempeñar el ferrocarril (Faith, 1990).

Los principales puertos y plataformas logísticas existentes no cuentan con conexiones directas ni de enlace internos (Morales, 2010). Resulta llamativo que el principal puerto de contenedores y pasajeros de España, Algeciras, quede casi aislado; sólo dispone de un débil enlace con el resto de vías interiores, hacia Madrid. Algo similar sucede a Almería y Cartagena. El cabotaje se presenta como una alternativa real y más ecológica (C.C.E. 2011, González, 2010). Casi todo está aún por hacer en tal sentido.

Muy recientemente la Alta Velocidad ferroviaria ha llegado al Mediterráneo. Procedente de Madrid, une Tarragona y Barcelona (acabada en 2008); también alcanza en Valencia (2010), mediante su enlace directo desde Madrid. Otros techos se encuentran en construcción en diferentes partes del Arco Mediterráneo. Pero, tales tramos no constituyen una realidad por el momento. Tampoco parece que ellos sólos puedan modificar sustancialmente las profundas carencias existentes.

Con esa débil e inconexa trama de infraestructuras ferroviarias se explica y entiende el uso reducido del ferrocarril. En el caso de pasajeros sólo circula un número mayor de trenes diarios en la parte septentrional del trazado. En torno a Barcelona, a los destinos de larga distancia se añaden los que desempeñan la función de mercancías.

Menor significación alcanza los que conectan con el Sur, hasta Valencia. También pueden asimilarse a esa categoría de cercanías los enlaces entre Murcia-Alicante. El tráfico de mercancías representa niveles muy inferiores a los volúmenes generados en el área, tanto de entradas como salidas. Además, la parte substancial de las mismas corresponde a los desplazamientos realizados entre las áreas de producción o tratamiento, ubicadas en espacios próximos al litoral y hacia el interior. Conviene destacar los numerosos efectos negativos derivados de este modelo de transporte (Julienne, 2002). Son de diferente naturaleza y apreciable envergadura. Todo esto ayuda a comprender el deseo de mejorar el ferrocarril, para cubrir las carencias existentes señaladas.

Los otros modos de transporte, marítimo y aéreo, por su propia naturaleza, participan de manera diferente en la accesibilidad y conexión en este ámbito mediterráneo. Ambos cuentan con destacados centros de movimiento de pasajeros y mercancías. Jalonan todo el Arco Mediterráneo español. Los datos del cuadro III resumen algunas magnitudes más significativas y permiten calibrar, con mayor detalle, su significación. 
Cuadro III - Transporte marítimo y aéreo, centros más destacados.

Table III - Maritime and air transport, main centres.

\begin{tabular}{lcc|lcc}
\hline & \multicolumn{2}{c|}{ Puertos, (2007) } & \multicolumn{2}{c}{ Transporte aéreo, principales aeropuertos, 2008 } \\
\hline \multicolumn{1}{c}{ Ciudades } & $\begin{array}{c}\text { Movimiento } \\
\text { (Mill. Tm.) }\end{array}$ & $\begin{array}{c}\text { Contenedores } \\
\text { (miles unidad.) }\end{array}$ & \multicolumn{1}{c}{ Ciudades } & $\begin{array}{c}\text { Pasajeros } \\
\text { (millones) }\end{array}$ & $\begin{array}{c}\text { Mercancías, } \\
\text { (Tm.) }\end{array}$ \\
\hline Cádiz, Bahía de & 7254 & 1331 & Jerez/Cádiz & 1530 & 81 \\
Cádiz, Algeciras & 74514 & 42398 & -- & -- & -- \\
Málaga & 6345 & 4071 & Málaga & 13547 & 5814 \\
Granada & -- & -- & Granada & 1447 & 72 \\
Almería/Motril & 9640 & -- & Almería & 1199 & 20 \\
Murcia/Cartagena & 24041 & 524 & Murcia/S Javier & 1994 & 2 \\
Alicante & 3651 & 1269 & Alicante & 9096 & 4532 \\
Valencia & 53593 & 32527 & Valencia & 5909 & 13329 \\
Castellón & 13148 & 1327 & Castellón & -- & -- \\
Tarragona & 36140 & 410 & Reus/Tarragona & 1294 & 11 \\
Barcelona & 51398 & 25432 & Barcelona & 32705 & 96433 \\
Gerona & -- & -- & Gerona & 4827 & 233 \\
\hline Suma & 279724 & 109289 & Suma & 73548 & 120527 \\
\hline Total España & 496860 & 139279 & Total España & 208517 & 607129 \\
\hline \% área/España & 56,29 & 78,46 & \% área/España & 35,27 & 19,85 \\
\hline
\end{tabular}

Fuente. Elaboración propia obre datos del Ministerio de Fomento

Las cifras son contundentes y precisan pocos comentarios; sólo se apuntan algunas referencias que perfilan sus rasgos más destacados. La figura 3, ayuda a comprender lo expuesto.

En lo referente al tráfico marítimo de mercancías cabe indicar:

a) Su movimiento total alcanza una cifra elevada, más de la mitad del conjunto español. Eso nos introduce en la significación económica del área y confirma su posición ventajosa de geolocalización; en especial cara a su porvenir, dentro de los grandes flujos de circulación mundial, sobre todo con Oriente. Todo lo que sea mejorar la vertebración interna de ese espacio, ampliará y reforzará su hinterland hasta enlazar con las principales áreas urbanas interiores españolas (Del Romero, 2008). No se olvide que este modo de transporte, es básico en las relaciones exteriores españolas; a su través se desplaza el $85 \%$ de nuestras importaciones y el $65 \%$ de las exportaciones.

b) Existe un número destacado de puertos en el Arco Mediterráneo. Lo cual conlleva una distancia media escasa, de uno a otro. Tal proximidad aumenta la competencia entre ellos. Ello puede dificultar la generación de economías de escala. Ahora bien, su jerarquía es marcada. Los cinco principales mueven 239,6 millones de Tm. Así mismo, esos cinco puertos significan casi la mitad del tráfico de todos los puertos españoles $(48,2 \%)$. Los tres primeros encabezan la jerarquía portuaria nacional. De nuevo, eso enfatiza la significación del ámbito mediterráneo español. 

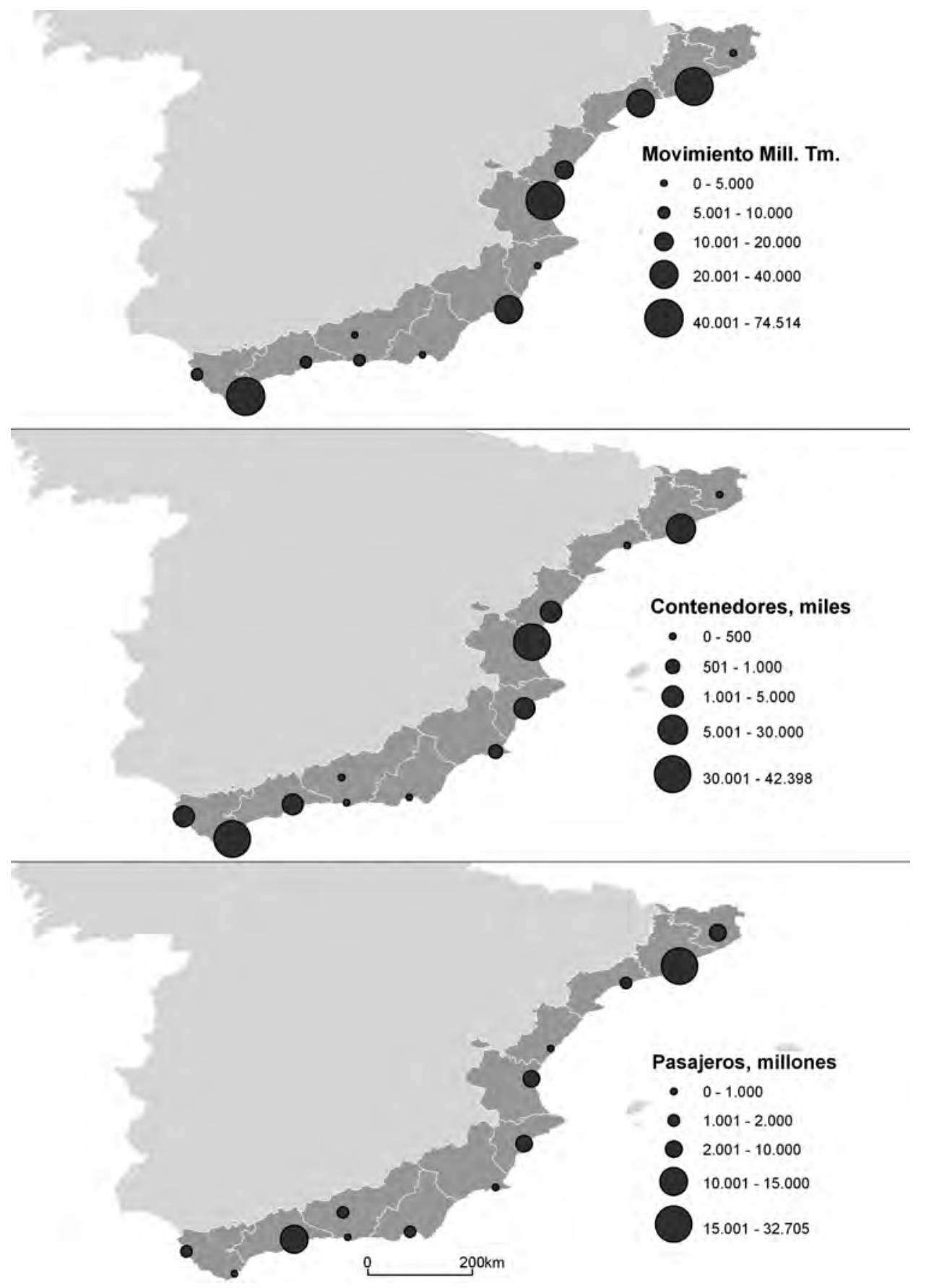

Fig. 3 - Significación del transporte marítimo y aéreo. Centros más importantes.

Fig. 3 - Significance of maritime and air transport. Major centers. 
c) El protagonismo destacado de estos centros de movimiento de mercancías debe contemplarse, cara al futuro, en su doble vertiente: ser nodos clave en la organización de las redes de transporte españolas y centros de gran dinamismo para la vertebración e integración del citado Arco, como área de atención preferente (Biehl, 1991).

Si nos circunscribimos al movimiento de contenedores, dado su protagonismo creciente en el tráfico de mercancías, su valor asciende. Por ellos (los puertos ubicados en el Arco Mediterráneo), pasaron en 2007, 109,2 millones de unidades; lo cual representa el 78,4 \% del conjunto español. Tales datos acentúan su preeminencia. Los tres principales puertos, Algeciras, Valencia y Barcelona son los de mayor trasiego español de estas unidades. La suma de su movimiento fue de 100,2 millones de unidades, es decir el 91,7 \% de la suma de todos los puertos mediterráneos y el 71,9 $\%$ de toda España. Prueba de su papel destacado como centros de movimiento de mercancías. Impulsar su dinamismo y facilitar su integración, constituye un horizonte deseable, que puede ayudar a la consolidación progresiva del Corredor Mediterráneo, junto al impulso añadido de sus mayores áreas urbanas (Graham, 2005). Pero no es menos cierto que para ampliar su hinterland, precisa mejorarse la adecuada vertebración del litoral con el interior Ibérico.

Los transportes aéreos, aunque con rasgos específicos y singulares, en referencia a lo aquí analizado, y atendiendo a los flujos de pasajeros, también confirman un dinamismo alto. La especialización turística del área ha favorecido ese devenir. No obstante, existe una acusada competencia interna poco favorable al conjunto.

Por esos aeropuertos pasaron en 2007 (último año antes de la actual crisis), 73,5 millones de pasajeros (el 35,2 \% de la suma de todos los aeropuertos españoles). Constituye una proporción algo inferior a la de su significación demográfica y económica. Atendiendo a los volúmenes de tráfico de pasajeros los aeropuertos muestran una jerarquía equilibrada. Barcelona, Málaga, Alicante y Valencia la encabezan. Sin embargo, en varios aeropuertos transitan reducidos volúmenes de pasajeros (entre uno y dos millones). Sin embargo, en lo concerniente al tráfico de mercancías, en general, su movimiento es insignificante. Representa una parte reducida de la suma nacional. $\mathrm{Su}$ recorrido futuro, por tanto, puede ser amplio. Barcelona concentra la mayor cifra.

Desde nuestra perspectiva de análisis, se desprende que este modo de transporte, confirma equipamientos sobrados. A través de ellos el Arco Mediterráneo español es un territorio con facilidades de comunicación. Otra cosa bien distinta es que las unidades de equipamiento estén bien dispuestas y gocen de un nivel de integración adecuada, capaz de contribuir a que los territorios que forman el Arco, a través del tráfico aéreo, contribuyan de manera suficiente a su consolidación posterior como Corredor. Más difícil aún es conocer su papel en un futuro Eje Mediterráneo.

Un balance conjunto de lo analizado, muestra una apreciable accesibilidad del Arco Mediterráneo español; si bien muy dispar en los diferentes modos de transporte. Abierto al exterior (sobre todo mediante el tráfico aéreo y marítimo). No obstante, la vertebración interna del mismo, muestra su apreciable compartimentación y una debilidad acusada mediante el modo ferroviario. Ahora bien, puede resultar insufi- 
ciente, preocuparse sólo de las mejoras internas, en especial, mejorando el modo de transporte ferroviario, sin hacerlo de forma paralela y complementaria buscando una mejor integración territorial con el conjunto español e ibérico: Eso se aborda a continuación.

\section{CORREDOR MEDITERRÁNEO Y VERTEBRACIÓN TERRITORIAL ESPAÑOLA, APROXIMACIÓN DESDE DIFERENTES ESCALAS}

Ya se ha apuntado que las infraestructuras de transporte existentes a lo largo del Arco Mediterráneo español, permiten hablar de un corredor de circulación incompleto; destacado, en el transporte de pasajeros, a través, sobre todo, de la carretera. Sus rasgos, características y capacidad muestran gradaciones descendentes acusadas de Norte a Sur. Por consiguiente, para alcanzar un Corredor de circulación que abarque a todo ese territorio y englobe otros modos de transporte, se precisan nuevas infraestructuras, lo cual precisa cuantiosas inversiones. Los programas de actuaciones contenidos en el Plan Director de Infraestructuras (MOPT, 1993), sólo se cumplieron parcialmente. Los más ambiciosos del Plan Estratégico de Infraestructuras (M. Fomento, 2004), en proceso de realización, distan de haber alcanzado aún los objetivos previstos. Los planes presentados recientemente (Marzo, 2011 y Febrero, de 2012), por dicho Ministerio, para completarlo (contando con previsibles y futuras ayudas europeas), proponen metas ambiciosas. Su horizonte de operatividad se fija en 2020. El coste de inversión se evalúa en 51.300 M€. Aquí se abarca el conjunto del corredor $(1.300 \mathrm{Km}$., de Algeciras a la frontera francesa). Pero, de nuevo, se contemplan mayores realizaciones en la parte Norte, la más equipada ya. Sus actuaciones se enmarcan en el proceso de revisión de las Redes Transeuropeas de Transporte (RTE-T) (Bliss et al., 1990; C.C.E., 2005). El objetivo es incluir dicho Corredor Mediterráneo como proyecto prioritario para Europa y destacado en España (C.C.E., 2010) (acuerdo de octubre de 2011). Se le adjudica el papel de corredor logístico de Europa. Conecta el Sur español con destino, en su parte más septentrional y oriental, en Estocolmo, Suecia y Zahony, Hungria; según propuestas planteadas hace años (Wackermann, 1995). Se busca, en especial, combinar la circulación terrestre (VRGC), de trenes de alta velocidad y convencionales (en su doble objetivo, tráfico de pasajeros y mercancías), circulando entre Algeciras y la frontera francesa.

Los objetivos del Ministerio de Fomento para el Corredor Mediterráneo son:

- Potenciar el rol de España como plataforma logística internacional.

- Contribuir al cambio del modelo productivo mejorando la calidad y fiabilidad del transporte de mercancías y reduciendo el coste por unidad transportada.

- Dotarlo de una red para el transporte de mercancías con capacidad suficiente.

- Asegurar su adecuada conexión con los grandes itinerarios de transporte de mercancías.

- Acondicionar, para el tráfico de mercancías, las líneas convencionales que puedan dedicarse a este tipo de transporte. 
Para eso es necesario acometer actuaciones en la red de Alta Velocidad, con mejoras en la red básica de mercancías. También conlleva realizaciones de naturaleza urbana, con mejoras logísticas, de acceso a puertos; etc.

De lo anterior se desprende que, dados los equipamientos existentes de carreteras, y como se ha apuntando ya, el modo ferroviario constituye la base central de las programaciones propuestas. Y, dentro de éste, el transporte de mercancías conforma su primacía. Parece como, si de pronto, se hubiese descubierto el déficit crónico y el descenso paulatino del ferrocarril, frente a la carretera, algo arrastrado desde más de cincuenta años, en el trasiego de mercancías (Calvo, 2006). Ahora, de manera repentina se busca modificarlo.

En consonancia con lo indicado, los propósitos específicos a conseguir, se focalizan en:

- Facilitar la circulación de trenes por las líneas de alta velocidad de tráfico mixto.

- Reducir las interferencias con los tráficos de viajeros.

- Consolidar una red básica de nodos e instalaciones logísticas eficiente, al completar los accesos ferroviarios.

- Promover la intermodalidad entre el ferrocarril y el transporte marítimo, a la vez que potenciar los accesos ferroviarios a los puertos.

- Aumentar la competitividad del transporte ferroviario de mercancías.

- Mejorar la gestión de la circulación de los trenes de mercancías y aumentar la cuota de participación en el transporte de mercancías.

Además de impulsar el tráfico de mercancías ferroviarias de manera central, también se contemplan algunas medidas relativas al transporte de viajeros, tales como:

- Asegurar las relaciones operativas fáciles con los corredores nacionales y europeos, incrementando la cuota de transporte

- Conectar y dar acceso a los grandes núcleos urbanos del corredor con una línea de Alta Velocidad para viajeros.

- Aumentar la alta velocidad comercial de los trenes, con tiempos de viaje más competitivos; con puntualidad y extrema fiabilidad.

- Mejorar el equipamiento y confort de las estaciones; también facilitando su acceso, dotándolas de estacionamientos e intercambiadores.

Tanto los objetivos como las propuestas son loables, fáciles de compartir. Pero, conducen a reflexionar sobre ciertas cuestiones de base, tales como:

i) Cualquier estudio acerca del Arco Mediterráneo, más aún si se hace desde una perspectiva dinámica, que contemple su cambio y transformación futura, debe enfocarse desde una escala territorial amplia (Gerondeau, 1996). Se aconseja que los planteamiento estratégicos españoles se combinen con el conjunto Ibérico, dentro del marco Comunitario Europeo (Serrano \& García, 2010). Desde el "lobby" de Ferrmed, junto a las propias CCAA mediterráneas, se argumenta y defiende la conveniencia de potenciar las infraestructuras, en especial las ferroviarias, para conseguir un auténtico Corredor, apoyándose en los datos socioeconómicos que contabiliza dicho 
ámbito, calibrando el potencial que encierra (Varios, 2011). Defienden sus intereses. A ello debe objetarse lo siguiente: el ya tradicional vacío de buena parte de la España central, y el desequilibrio a favor de algunas áreas mediterráneas, se agravaría con la potenciación primada del Corredor Mediterráneo, mediante infraestructuras de transporte más completas, flujos de tránsito más densos. Todo eso unido a una potenciación de su actividad productiva. Tal planteamiento inicial viene de años atrás (Arcarons \& Perellada, 1992). Pero, no parece que la consolidación de ese Eje Mediterráneo costero, frente a numerosas carencias centrales y septentrionales, sea una solución cabal que favorezca disminuir el desequilibrado territorio español, ni favorezca la armonía Ibérica (dejando de lado también los intereses portugueses).

ii) Conviene, así mismo, recordar un asunto clave. La grave crisis económica que atraviesa Europa, acentuada en sus países meridionales, es profunda y estructural. A menudo se apunta que, para salir de ella, se precisa articular un nuevo modelo económico. Dicho modelo debe crearse por completo, desde abajo. Eso invita a pensar que ese nuevo escenario debe sustentarse en otras bases que conlleven otra lógica de localización, frente a constantes territoriales existentes ahora, acaso válidas en el pasado, pero dudoso que sean útiles en el futuro (Capel, 2007). En un marco Ibérico, numerosas ciudades interiores y litorales de otros mares (Atlántico y Cantábrico), que padecen cierta atonía, ahora podrían ser focos de renovación e impulso en ese nuevo marco de renovación y cambio (Gutiérrez et al., 2006). Así mismo, semejante modelo productivo e innovador tal vez no precise del mismo nivel de infraestructuras convencionales, pensadas en los equipamientos actuales. Además, de poco valdrán copiosas inversiones en transportes, si no se acometen profundas transformaciones estructurales en el sistema productivo empresarial y acusados cambios en la política económica; ambos aspectos muy necesarios.

iii) Continuar apostando por costosas inversiones en infraestructuras, que completen los trazados existentes, muy débiles en la parte Sur del Arco Mediterráneo, debe ser objeto de reflexión (La Caixa, 2007); en especial, los ferrocarriles de Alta Velocidad, dados sus elevados costes económicos de construcción y funcionamiento (Campos et al., 2009). Se aconseja no emprender obras de infraestructura que sean un fin en sí mismo, en vez de sólo un medio, para alcanzar un beneficio posterior más útil. De lo contrario, seguiremos repitiendo el modelo anterior: inversión en infraestructuras, como motor económico básico, algo insuficiente y con profundas debilidades a largo plazo. Sus consecuencias favorables se agotan pronto, después se padecen sus consecuencias negativas.

En ese contexto, acaso también deben revisarse ciertas exposiciones críticas sobre el modelo territorial hispano, referidas al "centralismo español", proponiendo como alternativa un protagonismo mediterráneo (Bel, 2011). En algunos casos se 
impulsan desde la óptica de determinada región; la cual, de hecho, sólo defiende sus intereses, aunque sean más desequilibradores para el conjunto Ibérico.

Apostar de manera intensa por el ferrocarril conlleva disminuir la prioridad de la carretera. Ello tiene su clara lógica compensadora, ya mencionada. Es cierto que aquella está amenazada de saturación inmediata y va unida a numerosos inconvenientes (Krakovitch, 1996). Pero, por ahora, el transporte de mercancías en tren resulta el cincuenta por ciento más caro que por carretera. Además de las inversiones señaladas en ese campo, es necesario liberalizar el mercado, introducir la competencia y mejorar la gestión (Nastagi, 2003). De otra forma, lo dedicado al ferrocarril no será tan rentable por sí mismo. Se precisa conseguir un transporte de mercancías que realmente permita a las empresas ahorrar costes e incrementar su eficiencia. De lo contrario, de poco valdrán tantas inversiones, por sí solas.

En su conjunto, si bien no es sencillo, como se ha apuntado antes, es necesario, en cualquier planteamiento de realizaciones futuras, hacerlo desde la perspectiva que proporciona una escala territorial amplia, la que engloba al conjunto español e Ibérico, entroncado en el marco europeo; al menos en su dimensión occidental (C.C.E., 2002). Pues la realidad es compleja; pero unitaria.

Todo planteamiento de transporte que abarque un territorio de cierta envergadura, como éste, debe hacerse a escala Comunitaria. En el presente, y más aún en un horizonte temporal medio, se entiende que la Unión Europea, en lo que concierne a los transportes terrestres, precisa definir y articular unos corredores transeuropeos que permitan circular, personas, y mercancías, de un extremo a otro de su territorio, de manera cómoda y en cantidad elevada (Rus, 2006). La meta es vertebrar mejor el conjunto europeo y abrirlo más al exterior (C.C.E., 2008). Pero, ello debe hacerse sin acentuar los desequilibrios territoriales existentes en el conjunto de la península Ibérica. Desde hace años se ha ido trabajando con ese objetivo (C.C.E., 1990; 2007). Ahora bien, la morfología del territorio europeo dificulta esa tarea. La propia fisonomía Ibérica, con sus distancias alargadas impone ciertos condicionantes de trazado, los cuales no deben ignorarse (Bavoux y Charrier, 1994). A menudo no resulta sencillo escoger entre varias alternativas posibles. También, los intereses interestatales difieren; incluso, se producen desacuerdos (Bauchet, 1996). Es obvio que, en buena medida, su selección implica ventajas directas e indirectas, de dispar naturaleza, para unos e inconvenientes para otros territorios; las cuales van desde la subvención económica en las inversiones de infraestructuras a las consecuencias derivadas de su progresiva consolidación. De esa forma, la competencia de intereses locales y territoriales se acentúan (González y Benedicto, 2006). Eso sucede en este caso.

$\mathrm{Al}$ analizar una cartografía del conjunto europeo destacan varios aspectos. El carácter macizo de la Península Ibérica, y su localización alejada y extrema en el SE Comunitario determina un contacto de unión, limitado y montañoso con el país de engarce septentrional, Francia (Zembri, 2005). Sobre esa morfología territorial, la literatura científica al respecto, en sus planteamientos más detallados, contempla diferentes opciones básicas. En referencia al conjunto Ibérico, todas confluyen en su paso por los Pirineos. Ahí concurren intereses, a veces discordantes, entre españoles, 
portugueses y franceses (C.C.E., 2009). Previamente, las opciones diversas se circunscriben a su disposición y recorrido a través de la Península Ibérica, antes de llegar a los Pirineos.

De esa forma, la opción del tercer paso por los Pirineos centrales, en su discurrir por la provincia de Huesca, se ha descartado por el momento. Esto conlleva mantener amplios espacios españoles en clara situación de desenclave (todo Aragón). Sin duda el escaso interés de Francia en ello ha influido en la decisión final. Pero, eso no beneficia al conjunto Ibérico.

Escala Ibérica. No es fácil combinar las estrategias territoriales de ambos países. Cualquier planteamiento de tales asuntos dentro de los modos de transporte terrestre. El "espacio vital portugués" y sus prioridades se centran en la franja litoral occidental. Desde Oporto a Lisboa, en especial, con ciertas prolongaciones en otras direcciones. Combinar tal disposición para enlazarla con el resto de la Unión, a través de España, condiciona los trazados hispanos. Si se prima desde España la opción mediterránea, se marginan parcialmente sus intereses. La morfología de la España peninsular, la trama básica de su red urbana y sus espacios principales de producción organizan una realidad compleja (Gutiérrez, et al., 1992; Gutiérrez, 2004). La configuración de un modelo radial en la disposición de los principales ejes de transporte, se combina con contrastes acusados entre los vacíos centrales y las franjas litorales más pobladas; algunas de éstas, en especial las localizadas en su parte septentrional, quedan en franco desenclave frente a una potenciación de las áreas mediterráneas.

Los trazados, dentro de la parte española peninsular (compacta y maciza), al conectar los extremos opuestos, confluyen en el centro. Esa equidistancia hacia las áreas litorales, sin duda influyó en la elección política capitalina de Madrid hace siglos. Al paso de los siglos se ha consolidado ese modelo, reforzándose la radialidad de las conexiones (Romero, 2007).

Por otro lado, durante los últimos dos siglos se ha afianzado una tendencia acusada de aumento de los contrastes territoriales. Frente a unos espacios centrales que se despueblan, pierden peso y concentran sus efectivos, humanos y económicos, en unos pocos centros urbanos, destacando en extremo la "región urbana madrileña", otras regiones litorales adquieren mayor vitalidad. El nivel de accesibilidad ha tenido bastante que ver en ello. Pero hoy es una realidad indiscutible que, ese ámbito madrileño, con más de seis millones de personas, se ha convertido en la mayor AU de toda España y la que organiza con nitidez el conjunto del subsistema urbano español; es el principal nodo urbano, económico y funcional ibérico (Gutiérrez \& García, 2010).

De ahí se desprende que, cualquier trazado de eje básico transeuropeo Ibérico que soslaye Madrid, o no contemple su papel relevante, y posibilite su difusión mediata, no parece una opción realista, ni óptima cara al futuro. Indirectamente se dificulta la recuperación del amplio espacio central peninsular (Ureña et al., 2006). El extenso interior español, sus áreas septentrionales y occidentales, y su devenir futuro, aconsejan evitar operaciones que dificulten su devenir.

Todo ello no debe ser óbice para reconocer los beneficios de mejorar el Corredor de transporte Mediterráneo, que sin duda, dadas sus características, podría ser un 
impulso para la consolidación de un futuro Eje. La reciente selección e incorporación del citado Corredor, primado en su parte Norte, se ha celebrado de manera entusiasta, en especial por las regiones mediterráneas. Acaso se piensa, sobre todo, en sus ventajas de inversión directa e inmediata. Algunos hablan de una "nueva época" con lo cual se inicia el declive de la concepción del modelo radial y centralista de transporte. Frente a eso, se dice, se emprende el camino hacia un nuevo modelo mallado (Serrano, 2007). Hay dudas sobre ello, a la vez que se abren numerosos interrogantes.

\section{CONCLUSIONES}

El análisis de un tema tan complejo y vasto no es oportuno acabarlo con conclusiones cerradas. Eso puede ser pretencioso. No obstante, acaso es conveniente finalizar esta investigación con unas consideraciones finales, a modo de reflexión:

$1^{\text {a) }}$ Todo planeamiento territorial, que aborda un país de la dimensión, demográfica y económica como España, bien merece pensarlo y plantearlo, con visión de largo plazo, cara a sus estrategias futuras. Éstas deben ser bien meditadas teniendo en cuenta no sólo su horizonte próximo, sino también el de largo plazo. Más, en un tiempo de acelerados cambios mundiales, donde la escala de análisis debe ser global. España y toda la Península Ibérica, sólo conforman un eslabón reducido, débil, dentro del contexto Comunitario y Europeo. Por ello, más que nada, ese futuro Eje Mediterráneo debe calibrarse, como un camino, mediante el cual aprovechar las potencialidades reales y conjuntas de los dos países Ibéricos.

$2^{a}$ ) Las ventajas concurrentes previstas en el Corredor Mediterráneo español son destacadas. Unas, tal vez, pueden conllevar mayor incidencia directa; más favorable quizás, para las regiones directamente implicadas, dentro del devenir inmediato, en el marco del conjunto europeo. Se orientarían, sobre todo, cara a favorecer su geoestrategia de relación creciente con el pujante mundo oriental asiático. Otras, más próximas, representan, en especial, un impulso cercano para las regiones de la parte Norte del Arco Mediterráneo español. Pero, se abren numerosos interrogantes, desde los programas presentados a lo que puede ser, en el futuro, una realidad tangible, efectiva y real.

$3^{\text {a }) ~ P e r o, ~ s i ~ s e ~ p r i m a n ~ e n ~ e x c e s o ~ l a s ~ a ́ r e a s ~ m e d i t e r r a ́ n e a s, ~ s o b r e ~ t o d o ~ d e ~ s u s ~ r e-~}$ giones más septentrionales, el Sur y el centro Ibérico, acaso incrementen su aislamiento, quedando más descolgados. Se puede dificultar su integración y menoscabar el futuro impulso económico de amplias áreas Ibéricas. Los contrastes territoriales de toda la península tal vez aumenten de esa forma. Un asunto complejo a considerar y sobre el que debe reflexionarse de manera pausada; en especial, ante el escenario de cambio que precisa el nuevo modelo productivo que es necesario emprender en España, en Portugal y en buena parte de la Unión Europea. De lo contrario, junto a ciertos beneficios inmediatos, derivados de esos programas de actuaciones, también pueden derivarse ciertas consecuencias menos favorables para todos. 


\section{AGRADECIMIENTOS}

Agradezco la labor constante, en silencio y anónima, que llevan a cabo los revisores científicos en toda publicación. Merced a ello es posible la mejora del trabajo realizado.

\section{BIBLIOGRAFIA}

Auphan E (1997) Le transport ferroviaire. In Marcadons J, et al. (ed.) Les transports. Armand Colin, Paris: 88-116.

Bauchet P (1996) Les transports en Europe. La trop lente intégration. Economica. Paris.

Bavoux J J, Charrier J B (1994) Transport et structuration de l'espace dans l'Union européenne. Masson. Paris.

Biehl D (1991) The role of infrastructure in regional development. Infrastructure and Regional Development. Vickermann, R. (ed.): European Research in Regional Science, 1: 9-35.

Bel G (2011) España, capital París. Origen y apoteosis del Estado radial: del Madrid sede cortesana a la "capital total". Destino. Barcelona.

Bellet C, Alonso P, Casellas A (2010) Infraestructuras de transporte terrestre y territorio. Los efectos estructurantes de la llegada del tren de alta velocidad en España. Boletín de la $A G E$, 52: 143-163.

Calvo Soria J (2006) La política ferroviaria de la Unión Europea, 1953-2003. Universidad Rey Juan Carlos. Madrid.

Capel H (2007) Ferrocarril, territorio y ciudades. Biblio $3 w$. Revista bibliográfica de Ciencias Sociales, XII(717): 121-133.

COMISIÓN DE LAS COMUNIDADES EUROPEAS (C.C.E.) (2011) Maritime transport policy. Improving the Competitiveness, safety and security of European Shipping. Directorate General for Energy and Transport. Bruxels.

COMISION DE LAS COMUNIDADES EUROPEAS (C.C.E.) (1990) The Trans-European Networks, Bruxels.

Cuadrado Roura J R (1992) España en el marco económico y territorial europeo. Papeles de Economía Española, CECA, 11: 52-69.

Chesnais M (1997) Le transport, élément d'une stratégie complexe. Les transports, París: 7-35.

Del Romero L (2008) Planificació i govern del territori a l'arc mediterrani espanyol. Publicacions de l'Universitat de València. Est. y Doc., Valencia, $n^{\circ} 3$.
Faith N (1990) The world the railways made. The Bodley Head. Londres.

Fernández Guerrero J I, García Meéndez L, Suárez Burguet C (1992) Actividad exportadora y vertebración del Arco Mediterráneo. Papeles de Economía Española, Funcas, 11: 372-382.

Gerondeau Ch. (1996) Les transports en Europe. EDS Editeur. París.

González Vallvé J L, Benedicto Solsona M A (2006) La mayor operación de solidaridad de la Historia. Crónica de la Política Regional de la Unión Europea en España. Comisión Europea. Luxemburgo.

Gutiérrez Puebla J, García Palomares J C (2010) Procesos de descentralización de la actividad del Área Metropolitana de Madrid. In Feria $\mathrm{M}^{\mathrm{a}}$, Albertos J M (coordinadores) La ciudad metropolitana en España: procesos urbanos en los inicios del siglo XXI. Civitas, Thomson, Reuters. Pamplona: 377-407.

Gutiérrez Puebla J, Gómez C, García Palomares J C, López E (2006) Análisis de los efectos de las infraestructuras de transporte sobre la accesibilidad y la cohesión regional. Estudios de Construcción y Transporte, 105: 215-240.

Gutiérrez Puebla J, Monzon A, Piñero J M (1992) Accesibilidad a los centros de actividad económica en España. Dirección General de Planificación Interregional de Grandes Infraestructuras. M. O. P y Transportes. Madrid.

Julienne Ch. (2002) Le rail \& la route. Pour une nouvelle économie des transports. Les Belles Lettes. Paris.

Krakovitch A (1996) Prévision de la saturation des infrastructures routières en Europe après l'an 2000. Rapport, Union Européenne. Estrasburgo.

LA CAIXA (2007) Las infraestructuras. Informe mensual (V). Servicio de Estudios, Barcelona.

Merenne E (1995) Géographie des transports. Nathan, Paris.

Merlin P (1991) Géographie, économie et planification des transports. P.U.F. París. 
MINISTERIO DE FOMENTO (2004) Plan estratégico de infraestructuras y transportes. Secretaría de Estado de Infraestructuras y Transportes. Madrid.

MOPT (1993) Plan director de infraestructuras (1993-2007). Secretaría General Técnica, $2^{\mathrm{a}}$ ed. (edición amplia). Madrid.

Morales Gil A (2010) Las superficies logísticas y la organización espacial de redes de transporte de mercancías en España. Papeles de Geografia, 51-52: 211-222.

Nastagi Mattoni N (2003) Accesibilidad viaria y desarrollo territorial en la España peninsular: una propuesta de análisis. Geographicalia, 43, 81-96.

Pedreño A (1988) Un eje de expansión económica: Cataluña-Mediterráneo. In García Delgado J L (ed.) España-Economía, Madrid: 797-827.

Rodríguez J P, Comtois C, Slack B (2006) The geography of transport system. Routledge. Londres.
Romero J (2007) Geopolítica y gobierno del territorio en España. Editorial Tirant Lo Blanch, Valencia.

Rus Mendoza G de (dir.) (2006) La política de transporte europea. El papel del análisis económico. Fundación BBVA. Madrid.

Seguí Pons J Ma, Martínez Reynés M $\mathrm{M}^{\mathrm{a}} \mathrm{R}$ (2004) Geografia de los transportes. Universidad de las Islas Baleares. Palma de Mallorca.

Ureña J Ma , Garmendia M, Coronado J M (2006) Nuevos procesos de metropolización facilitados por la alta velocidad ferroviaria. Ciudady Territorio-Estudios Territoriales, 160: 213232.

Wackermann G (1995) Le transport de marchandises dans l'Europe de demain. Le défi de l'équilibre. La Recherche, Midi Éditeur. Paris.

Zembri P (2005) El TGV, la red ferroviaria y el territorio en Francia. Infraestructuras y Territorio, 70: 12-19. 